\title{
La dimensión política del I Festival Latinoamericano de Teatro (Córdoba, Argentina -1984)
}

\section{María Verónica Basile}

Centro de Investigaciones “María Saleme de Burnichon” (CIFFYH) Facultad de Filosofía y

Humanidades- Universidad Nacional de Córdoba

mvbasile@gmail.com

\author{
Verónica del Valle Heredia \\ Centro de Investigaciones “María Saleme de Burnichon” (CIFFYH) Facultad de Filosofía y \\ Humanidades- Universidad Nacional de Córdoba \\ vero_heredia_78@hotmail.com
}

Fecha de recepción: 21/06/2018. Fecha de aceptación: 09/09/2018.

\section{Resumen}

En 1984, la ciudad de Córdoba fue sede y productora del primer Festival Latinoamericano de Teatro interpretado por muchos como una manifestación políticocultural que trascendió el campo teatral. Oficializado por decreto provincial, buscó enmarcarse dentro de cierto clima eufórico que pretendía dejar atrás el pasado autoritario, destacando aquellos aspectos posibles a partir de la recuperación de la democracia, tales como la libertad de expresión, la calle - la ocupación del espacio público y la participación de quienes volvían del exilio, entre otros. Entre sus objetivos estaba además lograr la apertura de Córdoba hacia el mundo y, sobre todo, hacia Latinoamérica, haciendo fuertes referencias a la identidad e integración regional convocando a la participación de compañías y grupos teatrales latinoamericanos y europeos. En las siguientes líneas se propone una reflexión sobre las prácticas artísticas y culturales en el contexto de la restauración democrática, particularmente la experiencia del I Festival Latinoamericano de Teatro (FLT). Nos proponemos analizar los alcances que tuvieron algunas de las obras teatrales que formaron parte de la grilla oficial (Accions por La Fura dels Baus de España y la conocida como ópera rock El espectáculo va a comenzar de Córdoba, Argentina), como así también dos experiencias callejeras vinculadas a la denominada muestra paralela. Para ello, se privilegia un enfoque que recupera los aportes de la historia reciente y cultural y un corpus de análisis conformado por fuentes hemerográficas y documentales de la época.

Palabras clave

festival historia cultural pasado reciente teatro latinoamericano recuperación democrática 


\section{The political dimension of the First Latin American Theater Festival (Córdoba, Argentina -1984)}

\begin{abstract}
In 1984, the city of Córdoba hosted and produced the first Latin American Theater Festival, interpreted by many people as a political-cultural manifestation that transcended the theatrical field. Made official by the local government, it was associated with a euphoric climate that hoped to leave behind the authoritarian past, and thus the festival highlighted the aspects that were possible as a result of the recovery of democracy, such as freedom, the occupation of public space and the participation of those who returned from exile. One of its objectives was also to achieve the opening of Córdoba to the world and, above all, to Latin America, making strong references to identity and regional integration. This article examines artistic and cultural practices in the context of democratic restoration, particularly the experience of the first Latin American Theater Festival (I Festival Latinoamericano de Teatro-FLT). We analyze some plays that were part of the official grid (Accions by La Fura dels Baus of Spain and the well-known rock opera El espectáculo va a comenzar from Córdoba, Argentina) as well as two street experiences linked to parallel grid. We use an approach that recovers the contributions of recent and cultural history and a corpus based on press and documentary sources.
\end{abstract}

El primer Festival Latinoamericano de Teatro (IFLT) que tuvo lugar en Córdoba en el mes de octubre de 1984 trascendió lo artístico cultural para transformarse en un acto político que, con el tiempo, para algunos, fue convirtiéndose en un símbolo de festejo de la democracia restaurada. ${ }^{1}$ ¿Por qué el teatro es el elegido para lo que fue considerado por las fuentes epocales como la primera fiesta cultural en democracia? Puede reconocerse que Córdoba contaba con una fuerte tradición teatral que se remonta a la década del cincuenta con la creación del elenco provincial, la cual es continuada con directores que se fueron convirtieron en referentes de la actividad (Domingo Lo Giudice, Carlos Giménez, Jorge Petraglia). A partir de la década del setenta se dio un proceso en donde se profundizó el teatro político y surgieron nuevos espacios, lenguajes y estéticas (Arce, 2007). Si bien la actividad teatral había sufrido la censura impuesta por las autoridades durante el proceso dictatorial, ciertos grupos se mantuvieron activos en los márgenes del circuito oficial. Además, puede señalarse en términos contextuales la experiencia inmediata de Teatro Abierto.3 Tal continuidad permitió que una vez retomada la democracia, hubiera una red en funcionamiento que posibilitó y fue funcional al desarrollo del evento oficial propuesto por el gobierno provincial.

La mencionada tradición teatral cordobesa y sus hacedores fueron constantemente recuperados en los discursos de los referentes del teatro local:

Habíamos inventado el título del "nuevo teatro cordobés". Yo diría que los años “68 y”69 fueron nuestro "Woodstock". Nosotros veíamos un "Esperando a Godot" que no nos resultaba interesante si se hacía como un intento de copia clásica; hacíamos todo a la cordobesa (...) Traíamos gente de Buenos Aires para que viera el teatro de Córdoba, como a Adelach para que nos diera un seminario (Alberto Minero. Entrevista en Moll, Pinus, Flores. 1994).

Yo la identifico con que Córdoba aparte del Cordobazo y la Reforma del 18 tuvo la tercera pata de algo que es histórico, que la cultura por ser algo más que se

\section{Keywords}

Festival

Cultural History

Recent Past

Theatre

Democracy
1. En adelante utilizaremos las siglas IFLT para referirnos al Festival Latinoamericano de Teatro. Este fue promovido durante el gobierno provincial de la UCR a cargo de Eduardo C. Angeloz, quien a los pocos meses de asumir le otorgó por decreto su carácter oficial. Una primera aproximación sobre el Festival fue abordado en: Basile, M. V. \& Heredia, V.: “ Festival Latinoamericano de Teatro: escenas de la democracia recuperada". En Revista Afuera. Estudios de crítica cultural №15. 2015 Disponible en: http://www.revistaafuera.com/ articulo.php?id=342\&nro $=15$. 
territorializa distinto, que no es tan puntual si vos queres remitirnos a movimientos [como] el cuarteto [...] la cultura en Córdoba es más bien un fenómeno subversivo en sí mismo. Yo nunca me olvido por ejemplo en un tiempo cuando Petraglia estaba como director estable en la Comedia Cordobesa, antes de Cemento y del Parakultural en Bs As, él decía la cultura de Córdoba, el teatro de Córdoba es el más dinámico del país. (Arce, 2014, testimonio recuperado por Basile, Bruno \& Heredia)

El I FLT resignifica toda esa tradición teatral local en el contexto democrático que se vivía. El hecho de contar con una estructura previa y con fuertes referentes de la actividad, como artistas, grupos, espacios, permitió a las autoridades contar con un campo consolidado y hacer uso del mismo, con el fin de hacer visible en la sociedad el nuevo clima democrático. El festival era descripto como una iniciativa conjunta entre referentes del sector teatral y el gobierno provincial. Este último, parecía querer impregnarle y apropiarse de ciertos elementos presentes en este evento cultural. En ese sentido, a lo largo de las siguientes líneas, se pretende analizar aquellas características del IFLT que fueron asociadas con el retorno de la democracia tales como la libertad de expresión, el volver a habitar los lugares públicos, a representar lo que había sido ocultado/censurado bajo la dictadura y la recuperación de una identidad latinoamericana. Asignarle en el nombre el carácter y alcance "Latinoamericano" parecía explicarse, por un lado, por reconocer como a uno de sus creadores a Carlos Giménez quien, a su vez, desde el exilio había fundado el Festival de Teatro en Caracas (Venezuela). Por otra parte, esta denominación recupera el camino transitado por muchos referentes teatrales que ante la censura y la persecución hallaron refugio y un modo de continuar desarrollando la actividad en otros países de la región. Para muchos, el viaje era considerado como parte de una práctica política de búsqueda de una identidad latinoamericana. En otro sentido, parecía articularse con cierta intención del gobierno por restablecer una nueva mirada hacia la realidad del continente, oponiéndose así a las relaciones con Europa y Estados Unidos, que se habían priorizado durante el gobierno de facto. Por su parte, el catálogo oficial del festival señalaba: "resulta imperativo contactarse con la realidad de América Latina, la cual tan solo puede ser reflejada en su esencia mediante la "re-presentación" artística que, sin negar las coyunturas que configuran un producto, la muestran más allá de cualquier contingencia" (Catálogo IFLT, 1984, s/p.). Entre los elencos participantes pueden mencionarse que la gran mayoría eran provenientes de países de la región. ${ }^{2}$ Los grupos brindaban una diversidad de temáticas y lenguajes en sus obras, reconociéndose además el abordaje de algunas de las problemáticas y realidades compartidas en el subcontinente. Las obras analizadas en las siguientes líneas tienen en común que procedían del teatro independiente y la temática estaba relacionada con la historia y pasado reciente latinoamericano: dictadura, persecución política, exilio, desaparecidos, encarcelamiento.

Con el fin de aproximarnos a los contenidos y alcances de algunas de esas representaciones de aquel primer festival, haremos una reseña de obras que participaron en la muestra oficial y la paralela. Aunque no exclusivamente, seguimos a $\mathrm{H}$. Becker (2008), de quien tomamos algunas herramientas analíticas. Partiendo del concepto mundo del arte que, vertebra su enfoque y refiere a una red de personas cuya actividad cooperativa organizada produce una serie de trabajos que se definen como arte. La organización de esos mundos del arte articula a una red de productores, personal de apoyo, distribuidores y público. Asimismo, es posible por medio de convenciones, de la movilización de recursos, de la colaboración entre diferentes actores y del papel del Estado. En ese sentido, recuperamos para esta reflexión, la noción de convenciones, no sólo en relación a la producción, sino también respecto de los públicos. Para Becker, la cooperación es posible por medio de las convenciones, entendidas como "acuerdos previos que se hicieron habituales" respecto del modo de hacer las cosas en los mundos del arte. Estas "regulan las relaciones entre los artistas y el público,
2. La grilla oficial estuvo integrada por diecinueve grupos procedentes de Brasil, Colombia, Ecuador, México, Puerto Rico, Uruguay y Venezuela. Se incluye además España, que si bien puede cuestionarse por su pasado colonialista, en ese momento compartía un proceso de restauración institucional y además participa con un elenco de carácter disruptivo. Por otra parte la muestra paralela estaba compuesta por trece obras. Asimismo, durante el desarrollo del festival se fueron sumando otros grupos locales y nacionales. 
especificando los derechos y obligaciones de ambos"; configurando así su existencia" (cf. 2008: 48). Las convenciones generan distintos públicos. El autor distingue, un grupo de convenciones que forman parte de la cultura y que toda persona "socializada" conoce (cf. 2008:64). Señala que

Las convenciones que conocen todos los miembros bien socializados de una sociedad hacen posible algunas de las formas más básicas e importantes de cooperación que caracteriza a un mundo de arte (...) permiten que personas que tienen poca o ninguna familiaridad con el arte o la formación artística puedan integrarse a un público. (...) El conocimiento de estas convenciones define el perímetro de un mundo de arte e indica la existencia de posibles miembros del público de los que no cabe esperarse conocimiento especial alguno" (Becker 2008: 66).

Asimismo, advierte sobre otras que "solo conocen quienes están familiarizados con ese mundo del arte", a los que define como "miembros serios y experimentados" del público, que pertenecen y son parte más o menos permanente de la actividad cooperativa que lo constituye (cf. 2008: 68-69). Finalmente, se superpone a este grupo, los estudiantes de arte, personas formadas y semiformadas, que en ocasiones suele denominarse como "público educado". Para el sociólogo, son aquellos que conocen el oficio, las cuestiones técnicas y la utilización de habilidades porque "estuvieron del otro lado de la línea que separa a intérpretes y creadores de los consumidores" (cf. 2008:74).

\section{Escenas del festival}

En primer lugar, en términos contextuales, nos interesa señalar que pese al cierre del Departamento Universitario de Teatro durante los años setenta, continuaron funcionando la Comedia Cordobesa (elenco teatral oficial) junto al Seminario provincial de formación actoral Jolie Libois. Asimismo, como hemos señalado, puede reconocerse la permanencia de algunos de los referentes del circuito independiente, como así también un conjunto políticas vinculadas a la práctica teatral de carácter oficial durante el período dictatorial. Es decir, había en Córdoba un campo propicio para la emergencia del Festival, pero también un público formado y/o entendido, es decir, que compartía las convenciones, por esa tradición teatral local existente, conformado por hacedores independientes, profesionales y talleristas. No obstante, por otra parte, hubo obras que desafiaban de manera innovadora esas convenciones, en gran medida por el tipo de puesta y por el espacio en donde fueron llevadas a cabo que, a su vez, pese a ese carácter innovador, también buscaban alcanzar a ese público no especializado, más popular. Es decir, que hallamos los tres tipos de convenciones esgrimidas por Becker, aquellas que apuntan a materiales arraigados en la cultura, aquellas ligadas a la historia y el mundo teatral. Finalmente, respecto de los que, como mencionamos, recibieron una formación, pero que no necesariamente se dedicaron a las artes escénicas si bien compartían de manera cooperativa y/o conocían la dimensión técnica del oficio y de la producción teatral. Aunque no de manera estricta intentaremos ir dando cuenta de ellas en las siguientes líneas.

El espectáculo va a comenzar se encontraba entre-otras-como una de las obras que más repercusiones e interés despertó en el público de acuerdo a lo reseñado por la prensa. La obra se empezó a gestar entre 1982 y 1983. Su grupo Teatro Hoy se caracterizó por funcionar como un taller permanente. Se trataba de un grupo local perteneciente al circuito independiente que presentaba - con cierto carácter de novedad - un musical en formato de ópera rock, que, sin embargo, ya había sido estrenado durante los últimos años de la dictadura. Su director, Ricardo Sued, consagrado en el ambiente teatral local, afirmó que antes del IFLT tuvieron alrededor de ochenta presentaciones. Su contenido abordaba acciones vinculadas al terror, la represión, la falta de libertad, 
desapariciones y el papel de la Iglesia en aquel contexto autoritario (Moll, Pinus y Flores, 1996:175). Sued, consciente de que la obra tocaba temas que podían ser sensibles a las autoridades, manifestaba:

Fuimos quitando los velos de la censura y pusimos sobre el escenario nuestros miedos, nuestros dolores que son del hombre contemporáneo..., es una manera de demostrar que seguimos viviendo a través del teatro, aunque una generación haya desaparecido. En suma, ese es el mensaje: la vida contra la muerte (21 de octubre de 1984, La Voz del Interior, p. 2)

En la grilla oficial, El espectáculo va a comenzar representaba el aporte de Córdoba. ${ }^{3}$ La misma tuvo como sede el Centro Cultural General Paz, un ex mercado de abasto, que había sido refuncionalizado por el gobierno de facto como un polo barrial para el desarrollo de actividades culturales. En su carácter de ópera rock combinaba la danza, la música y el teatro. Pese algunas críticas sobre el relato dramatúrgico de la obra, el grupo Teatro Hoy es recordado por su participación, por el nivel de activismo político asumido al regresar la democracia. Durante el IFLT, una de sus funciones fue auspiciada por la Comisión de Familiares de Desaparecidos y Detenidos por razones políticas (25 de octubre de 1984, La Voz del Interior, p. 13). No obstante, no fue la única que asumió este compromiso.

Otra de las obras destacadas, a pesar de haber integrado la muestra paralela, fue Lucifer en el zoo. Su peculiaridad residía en ser representada por el grupo Escena, el cual estaba conformado por presidiarios integrantes del taller teatral que funcionaba en la Unidad Regional Número 4 de la ciudad cordobesa de Río Cuarto. La iniciativa, dirigida por el chileno Sergio Valencia, fue presentada en el IFLT como la primera de este tipo en Argentina. A partir de la recopilación de improvisaciones, el argumento aglutinaba las frustraciones y conflictos de aquellos privados de la libertad, como parte de una crítica social profunda. Los actores representaban situaciones - sueños y fantasías - en las que eran libres, pero que se veían frustradas. En una de las memorias oficiales que llevaba por título "Los diez días que conmovieron a Córdoba" se destaca la escena final en la que los hombres son animales tras las rejas del zoológico, resumiendo así con toda intensidad el drama del cautiverio y obligando no sólo al aplauso, sino también a la reflexión. (1984:59) 4 Por otra parte, fue considerado como algo atípico para un festival, la actuación que este grupo llevó adelante en la Penitenciaría del Barrio San Martin, uno de las cárceles más grandes de la ciudad. Las fuentes oficiales agregaban: "el espectáculo se convirtió de inmediato en un diálogo constante entre la sala y los actores. Lo que se narraba en el escenario no era para nada ajeno a la experiencia diaria de los reclusos que ocupaban la platea." El resto de las funciones de Escena continuaron en una de las Salas del Teatro San Martín, sede principal del IFLT.

La dimensión Latinoamericana del festival pudo evidenciarse no sólo en que la mayoría de los elencos pertenecía a los países de la región, sino también en sus obras. La delegación de Brasil llegó a la muestra oficial con la puesta en escena de Morte a os brancos, creación colectiva del grupo Uniao e Olho Vivo (que puede traducirse como Unidad y ojo atento). Definido como teatro popular, con tradición en la promoción de una conciencia crítica entre los sectores oprimidos, el grupo se caracterizó por su presentación en la periferia, en plazas, calles y teatros no convencionales de los barrios populares de Brasil..$^{5}$ La obra Morte a os brancos. A Lenda de Sepé Tiaraju hace referencia a la vida en las misiones jesuíticas durante 1759 y el intento por implantar allí una forma de vida comunitaria a través de una revuelta popular. Esto traerá persecuciones políticas y encarcelamientos para sus líderes guaraníes (19 de octubre de 1984, La Voz del Interior, p.13). Enfocada en un episodio trágico e histórico de resistencia cultural, la obra se pretende una crítica

3. En la grilla oficial además de Córdoba estuvieron representadas las provincias de Buenos Aires y Entre Ríos.

4. Cabe señalar es posible también reconocer la dimensión política y el carácter revolucionario, de cambio que se le atribuyó en términos simbólicos al festival, en la denominación misma de una de las memorias en la que se retoma el título del libro del periodista John Reed Ten Days that Shook the World en el que recoge y crónica su testimonio sobre la Revolución Rusa y que fuera traducido y editado para América Latina en 1959.

5. Véase LVI 26-08-1984, p.3 y Teatro Popular União e Olho Vivo. En: ENCICLOPÉDIA Itaú Cultural de Arte e Cultura Brasileiras. São Paulo: Itaú Cultural, 2017. ISBN: 978-85-7979-060-7 Disponible en: http://enciclopedia.itaucultural.org.br/grupo209049/ teatro-popular-uniao-e-olho-vivo 
sobre la conquista. No obstante, si bien estaba contextualizada en el período colonial, subyacía la intencionalidad de una lectura con ese pasado reciente latinoamericano. La puesta combinaba la actuación, la música, el baile y el diálogo con el público, lo cual, según la prensa, generaba muchas veces un impacto $\mathrm{y}$, a veces, desorientación entre los asistentes (22 de octubre de 1984, La Voz del Interior, p. 13) El grupo también tomó parte activa en distintas intervenciones políticas que fueron cronicadas por los medios. Fuera de la programación del festival, ofrecieron una función al finalizar una marcha de organismos defensores de los derechos humanos que pedían por la liberación de presos políticos y mostraron su solidaridad ante un grupo que realizaba una huelga de hambre por este motivo (19 de octubre de 1984, La Voz del Interior, p.13).

Como parte integrante de la grilla oficial, aunque no provenía de la región latinoamericana, sino de España, se destacó la participación de la Fura dels Baus (en adelante FDB). Llega a participar en el FLT con la obra Accions, siendo su director Andrés Morte, cofundador del grupo. En 1979, habían iniciado sus actividades como teatro de calle, algo que luego abandonarían, aunque manteniendo en sus puestas la idea de horizontalidad con el público. Se proponían explorar otras dimensiones del espacio y una interacción experiencial con el espectador. De acuerdo con sus propias memorias, Accions planteaba muchos de los elementos que luego consolidarían el lenguaje teatral de FDB. Sin un argumento definido, el espectáculo encadenaba siete acciones que combinaban la música y lo performático. En sus memorias sostienen que, aunque se iniciarán en un punto elevado, todas las acciones concluían desarrollándose al nivel del espectador, a ras del suelo y presentan un diálogo entre el ámbito arquitectónico, el público y las evoluciones de los actores. ${ }^{6}$ En Córdoba, el escenario elegido fue la ex Escuela E. Olmos que se encontraba clausurada hacía un tiempo (hoy devenida en shopping center). El edificio estaba localizado en una de las principales avenidas de la zona céntrica de la ciudad colindante con el Teatro Provincial San Martín. Las acciones se desarrollaron principalmente en el patio de aquellas instalaciones que se encontraban abandonadas, condición que creaba junto a otras, un lenguaje escénico particular que incluía la demolición de paredes, la introducción de objetos desagradables, la destrucción, entre ellos el desguazamiento de un automóvil, en el que, en el caso cordobés, por motivos económicos, de acuerdo con el testimonio de algunos asistentes, se utilizaron otros artefactos de consumo como televisores, heladeras, etc., siendo el propio público el que los destrozaba. En otros casos, según los testimonios y la prensa, la experiencia parecía ser de una extremidad ajena a las convenciones (Becker, 2008) del público teatral cordobés, que provocaba que algunos asistentes buscaran huir. Para Alex Ollé, uno de sus integrantes: "era Teatro Visceral. Queríamos agitar al público de espectáculos tradicionales. Crear fricción. Romper coraza (...) La interacción y el contacto físico que proponíamos era un acto catártico, vivencial." (Ospital, 2009:56) La obra fue presentada como aquella que rompía con las normas.

\section{...el espectador, sin otra salida, se ve forzado a defenderse de una violencia teatral llevada al límite del peligro. (...) "Accions" es la teatralización de un mundo sin piedad, la respuesta furiosa frente a la violencia cotidiana de nuestras sociedades “civilizadas"(Los diez días que conmovieron..., 1984: 38)}

Devenido en un hito del campo teatral, la presencia de la FDB en aquella primera edición del Festival forma parte de cierta memoria colectiva entre quienes asistieron por su lenguaje performativo y carácter rupturista y/o vanguardista, pero, sobre todo, por la experiencia que provocó en aquel momento tan próximo a un pasado traumático, como así también su permanencia en el contexto reciente. A veinticinco años de aquella presentación, una revista local recupera algunos de los testimonios, entre ellos el de Rafael Reyeros, uno de los organizadores del FLT:
6. Véase en La Fura dels Baus: https://www.arenateatro.info/espectaculos/teatro/adn-la-fura-dels-baus/ 
Uno de los hitos fueron esos fetos que se chocaban contra las telas. Y la sangre que corría por la avenida era muy fuerte porque la memoria de la Triple A seguía estando. Actions podía significar miles de cosas. Nuestra historia reciente recortaba el sentido para la gente que entonces se descubría envuelta por un código mayor, un lenguaje artístico que hablaba de la violencia más allá de nosotros: Los espectadores ayudaban a destruir y veían a esos tipos que salían de las catacumbas. Todo eso se reinterpretaba en función de lo que nos había pasado (Ospital, 2009:56).

En relación a la participación del FDB nos interesa, por último, señalar que, de acuerdo con sus testimonios de sus integrantes, Argentina se constituía en un referente artístico, en teatro y cine, pero, a su ve,z aquella primera presentación en Córdoba sería recuperada por algunos artistas locales como Marcelo Nusenovich y los postfunk Enviados del Señor, además de la porteña Organización Negra.

Otra dimensión del IFLT en la que nos interesa detenernos se refiere a la participación de quienes retornaron al país después de años de exilio, para formar parte de este evento cultural. Entre ellos puede mencionarse a Carlos Giménez (director del festival), Roberto Videla 7 , Paco Giménez ${ }^{8}$ y Graciela Ferrari, entre otros. Esta última regresa a Córdoba para integrar la muestra paralela del IFLT con Avevals, la tierra ninguna. Se trataba de un unipersonal que Ferrari comenzó a gestar a fines de 1981, en México, y estrenó en un festival de teatro de Verona (Italia), países en los que había vivido durante su exilio. La obra, que "habla de aquella parte de la humanidad que la suerte quiso provisoria, que vive de lugar en lugar deambulando por la tierra de nadie." (4 de octubre de 1984, La Voz del Interior, p.14), fue considerada una especie de relato en primera persona de la experiencia del exilio: "es más que una representación. Es el grito de quienes se fueron para sentirse extranjeros en cualquier parte (...) Historia personal y, a la vez, testimonio del drama de un pueblo castigado" (1984:57). La muestra paralela conjugó diversas experiencias en el marco de las actividades paralelas del festival que, a diferencia de la oficial, parecía ofrecer un contacto más directo e interactivo con el público.

Si bien algunos no adherían a la división en muestra oficial y paralela, por el peso que conllevan estas palabras, pueden señalarse algunas distinciones entre una y otra. La muestra oficial estuvo integrada por obras representadas por grupos con trayectoria internacional y cuyo contenido hicieran referencia a la problemática latinoamericana, aspectos estos que estaban dentro de los requisitos de la convocatoria al festival. Por su parte, la muestra paralela intento hacer un lugar en el IFLT a los grupos independiente, incluyendo a aquellas representaciones con puesta experimental, que fuesen originales y que "tratara de ocupar espacios libres y convertir a Córdoba en un inmenso escenario abierto a todos", combinando lo popular y lo experimental (5 de agosto de 1984, La Voz del Interior, p.3). Dentro de las dos secciones se aprecia que gran parte de las obras estaban atravesadas por las experiencias recientes de los países, grupos o actores. Asimismo, ambas se caracterizaron por la diversidad y la amplia convocatoria del público; sin embargo, la paralela, según las memorias del festival, "centraron su atención tanto en la renovación como el rescate." (1984:51) La principal distinción de la muestra paralela respecto de la oficial fue el teatro callejero: según la reseña había concretado y superado uno de los objetivos del festival, es decir, "Que el teatro invada la Ciudad." (Ibídem). Sin embargo, esta particularidad, que venía promoviendo el municipio y la Secretaría Provincial de la Juventud, no era tan ajena a prácticas teatrales que habían tenido lugar en Córdoba antes de la dictadura, más bien ligadas con lo que algunos denominan teatro popular y teatro político. A su vez, acompañaba ciertas políticas de descentralización que, paradójicamente, se habían iniciado durante el gobierno de facto, pero que cobraron fuerza con el retorno del régimen democrático y al discurso de federalización planteado por el gobierno nacional de igual signo partidario, vigente en la provincia y municipio. Por otra parte, en
7. Docente de teatro y cineasta. Integró el grupo Libre Teatro Libre. 8. Actor y director, integrante del grupo La Chispa. Desde 1984 dirige La Cochera, sala de teatro independiente. 
líneas generales, el festival rompía con los discursos centralistas, en sus diferentes planos; es decir, en relación a Córdoba y su interior provincial, respecto de la capital porteña, pero también de la región frente a las capitales culturales y económicas centrales y/o hegemónicas.

En cierta medida, el modo de producción y desarrollo del festival iban constituyéndose sobre bases y prácticas - formas de hacer democráticas que intentaban dejar atrás y oponerse al pasado autoritario reciente. En efecto, se trató de un acto político democrático, no sólo en términos partidarios o como forma de gobierno, sino en el sentido amplio y performativo de la palabra.

\section{Conclusiones}

En estas líneas nos interesó sobre todo reflexionar y destacar el diálogo de las obras con el pasado reciente y la construcción democrática, la interpelación y experimentación con el público y cierto carácter rupturistas respecto de sus puesta en escena, no sólo en lo técnico, sino en términos espaciales, en lo que algunos definen como no convencionales y un escenario mayor, que se convirtió la ciudad y diferentes lugares de ella: la calle, las plazas, la cárcel, etc.

Si bien este trabajo no se trató de un estudio sobre la recepción, recuperamos de las diversas fuentes, el alto grado de participación y cómo éste fue interpelado, que una de las reseñas oficiales destaca como algo singular y deposita en el público el carácter vanguardista. Esto pudo evidenciarse con su presencia en la calle, la asistencia y aceptación de obras que rompían con determinados lenguajes escénicos tradicionales, entre otros. En particular, suele asignarse a Córdoba un carácter clerical y conservador; sin embargo, como señalamos, no hubo oposiciones a lo que se estaba mostrando, sino parecía palparse según los diferentes relatos, un clima de efervescencia, libertad y alegría propia de un festival, que se ligaba más con los mitos de una Córdoba moderna, rebelde y universitaria.? Consideramos que muchos de los asistentes eran esos estudiantes a quienes, en el pasado reciente, les habían sido clausurados diferentes espacios de creación, como así también los ingresantes o aquellos que retornaban en busca de nuevos lenguajes, discursos, etc. Y, por ello, consideramos que el vínculo entre las obras y los espectadores, que trascendieron esa categoría participando espontáneamente y convirtiéndose también en productores del festival, estuvo dado por convenciones no sólo culturales por el hecho de compartir realidades y un pasado reciente traumático, sino también por el conocimiento especializado del mundo teatral y sus particularidades técnicas.

Si bien la consideración de sólo cuatro obras es una muestra pequeña y recortada de la totalidad del festival, da cuenta de la heterogeneidad y diversidad de los grupos participantes y de los lenguajes y recursos escénicos desplegados que, no obstante, coinciden en un contenido crítico que remite a cierta idea de ruptura y cambio en ese contexto histórico y respecto de las realidades de la región y el mundo.

Son muchos los planos y dimensiones susceptibles de análisis, que exceden a estas líneas, pero que hemos ido abordando en diferentes trabajos y de los cuáles se siguen abriendo interrogantes.

Reconocemos que si bien no se trató de un análisis exhaustivo de las obras, intentamos aproximaciones a las mismas, buscando revisarlas no en términos dramatúrgicos ni autónomos o aislados, sino más bien puestas en diálogo y en consideración de aquellos aspectos que remiten al mundo del arte, en este caso del teatro, siguiendo a Becker (2008), pero que su vez lo trascienden, reflexionando desde su dimensión histórica y cultural.

9. Sin ser textual o lineal a lo que allí se discute, nos remitimos a un debate existente en el campo historiográfico. 


\section{Q Bibliografía}

» ADN La Fura dels Baus (s/d) Disponible en https://www.arenateatro.info/espectaculos/teatro/adn-la-fura-dels-baus/

» Basile M. V. \& Heredia, V. (2015) “I Festival Latinoamericano de Teatro: escenas de la democracia recuperada", Revista Afuera. Estudios de Crítica Cultural (15), [en línea]. En: http://www.revistaafuera.com/articulo.php?id=342\&nro=15

» Basile, M. V. (2016) Políticas culturales municipales de Córdoba. Tesis de Doctoral en Estudios Sociales de América Latina, CEA - Universidad Nacional de Córdoba. (Inédita)

» Becker, Howard (2008) Los mundos del arte. Sociología del trabajo artístico, Buenos Aires: Universidad Nacional de Quilmes.

» González, S. \& Basile, V. coord. (2014) Juventudes, políticas culturales y prácticas artísticas. Fragmentos históricos sobre la década de 1980, Córdoba: Alción Editora

» Heredia, V. (2013). "Los Festivales Latinoamericanos de Teatro en Córdoba: escenarios de la democracia 1984-1994" (ponencia presentada en "I Congreso Internacional de Ciencias y Sociales Humanidades. Perspectivas y debates actuales a 30 años de la democracia", Córdoba) [en línea] En http://www.conferencias.unc.edu.ar/index.php/ponencias/ponencias2013.

» Moll, V., Pinus J. y Flores, M. (1996) Las lunas del teatro. Los hacedores del teatro independiente cordobés (1950-1990), Córdoba: Ediciones del Boulevard.

" Ospital, L. (2009) "A 25 años de la Fura dels Baus en Córdoba. Fue la hostia”, Revista La Central (10) pp. 54-57

» TEATRO Popular União e Olho Vivo. En: ENCICLOPÉDIA Itaú Cultural de Arte e Cultura Brasileiras. São Paulo: Itaú Cultural [en línea] en: http://enciclopedia. itaucultural.org.br/grupo209049/teatro-popular-uniao-e-olho-vivo

\section{Fuentes Hemerográfica}

» La Voz del Interior, 1984.

\section{Fuente documental}

" Catálogo Oficial I Festival Latinoamericano de Teatro (Córdoba, 1984).

»Los diez días que conmovieron a Córdoba (Córdoba: Centro de Documentación Artístico - Cultural, 1984). 